1

2

3

4

6

7

\section{How Codon Usage Bias Affects Our Ability to Recover the Tree of Life}

Justin B. Miller ${ }^{1}$, Michael F. Whiting ${ }^{1,2}$, John S.K. Kauwe ${ }^{1}$, Perry G. Ridge ${ }^{1, *}$

${ }^{1}$ Department of Biology, Brigham Young University, Provo, UT 84602, USA

${ }^{2}$ M.L. Bean Museum, Brigham Young University, Provo, UT 84602, USA

${ }^{*}$ Correspondence to be sent to: 4102 LSB, Department of Biology, Brigham Young University,

Provo, UT 84602, USA;

E-mail: perry.ridge@byu.edu 


\section{Abstract}

9 Phylogenies depict shared evolutionary patterns and structures on a tree topology, enabling the

10 identification of hierarchical and historical relationships. Recent analyses indicate that phylogenetic

11 signals extend beyond the primary structure of protein or DNA, and various aspects of codon usage

12 biases are phylogenetically conserved. Several functional biases exist within genes, including the number

13 of codons that are used, the position of the codons, and the overall nucleotide composition of the

14 genome. Codon usage biases can significantly affect transcription and translational efficiencies, leading

15 to differential gene expression. Although systematic codon usage biases originate from the overall GC

16 content of a species, ramp sequences, codon aversion, codon pairing, and tRNA competition also

17 significantly affect gene expression and are phylogenetically conserved. We review recent advances in

18 analyzing codon usage biases and their implications in phylogenomics. We first outline common

19 phylogenomic techniques. Next, we identify several codon usage biases and their effects on secondary

20 structure, gene expression, and implications in phylogenetics. Finally, we suggest how codon usage

21 biases can be included in phylogenomics. By incorporating various codon usage biases in common

22 phylogenomic algorithms, we propose that we can significantly improve tree inference. Since codon

23 usage biases have significant biological implications, they should be considered in conjunction with

24 other phylogenetic algorithms. 


\section{The Continued Importance of Phylogenetic Systematics}

27 Phylogenetic systematics explores the historical and hierarchical relationships among genes, individuals, populations, and taxa. Phylogenies allow biologists to infer similar characteristics in closely related species and provide an evolutionary framework for analyzing biological patterns (Soltis and Soltis 2003).

Furthermore, phylogenies are statements of homology and are used to organize shared structures or patterns between species (Haszprunar 1992). Originally, phylogenies were recovered using only morphological data. However, with the increased availability of molecular data, a combined approach using morphology and genetic markers is typically used in phylogenetic analyses (Bertolani, et al. 2014).

Although genetic data provide researchers with access to more species, it typically requires large amounts of data cleaning (e.g., alignment and annotation) before it becomes useful. Some of the greatest difficulties in recovering phylogenetic trees from molecular data (e.g., multiple substitutions at the same position between ancient terminal branches or no substitutions in a gene in short internal tree branches) are explored by Philippe, et al. (2011). These issues have recently become more pertinent as sequencing costs have dropped and genomic data now spans the Tree of Life.

\section{Codon Usage Biases Span the Tree of Life}

42 Codon usage bias is present throughout molecular datasets. There are 61 canonical codons plus three

43 stop codons that form and regulate the creation of 20 amino acids and the stop signal (Crick, et al.

1961). Since there are more codons than amino acids, the term synonymous codon is used to describe how multiple codons encode the same amino acid and were presumably identical in function. However, an unequal distribution of synonymous codons occurs within species, especially within highly expressed

47 genes, suggesting that synonymous codons might play different roles in species fitness (Sharp and Li 1986). Furthermore, an unequal distribution of tRNA anticodons directly coupling codons also varies 
50

51

52

53

54

\section{Overview of Common Phylogenomic Techniques}

58 Homologous sequence comparisons are commonly used to identify species relationships. Homologous

codon nucleotides during translation (Crick 1966). Codon usage is highly associated with the most abundant tRNA present in the cell (Post, et al. 1979) and codon usage patterns affect gene expression (Gutman and Hatfield 1989). Non-random mutations or selection for phenotypic differences caused by differential gene expression could explain some of the phylogenetic differences in synonymous codon usages. Although codon usages directly affect phenotypes, common phylogenomic approaches typically ignore the influence of codon bias in tree inference.

characters are identified by aligning orthologous genes and detecting character state changes of amino acid residues or nucleotides across a tree topology. This multi-step process is time-consuming and requires orthologous gene annotations. Non-homologous sequence comparisons have also been explored in alignment-free methods and will subsequently be discussed.

\section{Ortholog Identification}

Orthologs are genes within two or more species that usually share the same function because they are derived from the same ancestral gene in the most recent common ancestor (Koonin 2005). In contrast, paralogs may share the same function, but can arise from gene duplication or horizontal gene transfer. Paralogs may not be under the same evolutionary pressures and should not be compared in a direct positional alignment because these comparisons are a poor indicator of phylogenetic relationships (Koonin 2005). An in-depth evaluation of ortholog identification techniques is presented by Tekaia (2016). Once an ortholog is identified, phylogenetic studies typically require a multiple sequence alignment to align homologous characters. Reviews of some common multiple sequence aligners such as T-coffee (Magis, et al. 2014), MUSCLE (Edgar 2004), Clustal (Sievers and Higgins 2014), Clustal Omega 
74 (Sievers and Higgins 2018), and MAFFT (Katoh and Standley 2014) can be examined elsewhere

75 (Daugelaite, et al. 2013; Pais, et al. 2014).

76

77 2. Recovering the Phylogenetic Tree

i. Maximum Parsimony

79 Maximum parsimony assumes that each character is equally important and minimizes the number of

80

81

82

character state changes to recover the relatedness of species. Proponents of parsimony point to its explanatory power and ability to minimize ad hoc hypotheses (Farris 2008). However, parsimony can be misleading if unequal evolutionary rates between lineages exist because longer evolutionary branches have a tendency to form monophyletic groups even if the species have different phylogenetic histories (Felsenstein 1978). PAUP (Wilgenbusch and Swofford 2003) and TNT (Goloboff, et al. 2005) are two popular software packages to identify phylogenies based on parsimony.

\section{ii. Maximum Likelihood}

Maximum likelihood requires specific models of evolution that show the probability of character state changes and can be used in the likelihood function. Maximum likelihood calculates the probability of obtaining the data given the model and tree topology. One of the main reasons that maximum

likelihood estimates have gained traction is the mathematical property of consistency, which states that as more data (phylogenetically informative characters) are added, the likelihood function will converge to the correct tree (Wald 1949; Rogers 1997). Furthermore, maximum likelihood takes into account more complex modelling of datasets, and the modelling has become more computationally tractable through faster algorithmic design and faster computer processors (Paninski, et al. 2004). However, in exact opposition to maximum parsimony, maximum likelihood is more likely to separate highly divergent species, leading to long branch repulsion (Siddall 1998). MEGA X (Kumar, et al. 2018), RAxML 
98

99

100

101

102

103

104

105

106

107

108

109

110

111

112

113

114

115

116

117 phylogenetic reliability is difficult to ascertain from highly divergent sequences (Holder and Lewis 2003).

118 However, distance-based methods are frequently used when sequence alignments are not available or 119 in whole genome comparisons. Since genome assembly and multiple sequence alignment affect

120 phylogenies more than the technique used to recover the phylogeny, alignment-free methods attempt

121 to recover shared phylogenetic history without an alignment by comparing basic characteristics of

(Stamatakis 2014), IQ-TREE (Nguyen, et al. 2015) and PHYLIP (Retief 2000) are commonly used to recover phylogenies using maximum likelihood.

Bayesian phylogenetic estimates use posterior probabilities of a distribution of trees calculated with Markov Chain Monte Carlo (MCMC) techniques to evaluate tree probabilities. Bayesian inference adds statistical support to phylogenies and empirically produces more accurate trees in simulations.

However, Bayesian inference is highly sensitive to prior probabilities (Huelsenbeck, et al. 2002). How Bayesian techniques compare to other phylogenetic methods is addressed by Yang and Rannala (2012) and popular Bayesian techniques are implemented in MrBayes (Ronquist, et al. 2012; Ling, et al. 2016) and BEAST2 (Bouckaert, et al. 2014).

Distance-based phylogenies use techniques such as neighbor-joining to quickly produce relatively good trees and are often used as a starting point for phylogenetic analyses using other methods. Neighborjoining decomposes a star tree by taking the two closest taxa based on the number of character changes between them, pairing them together, recalculating weights based on the shortest distance between the paired species and all other species, and repeating this process until all taxa are paired. Although this technique is computationally fast, compressing the sequences into distances loses information and to recover shared phylogenetic history without an alignment by comparing basic characteristics of 
122 genomes (i.e., GC content, k-mer counts, codon usages, etc.) (Chan, et al. 2014). Broadly, alignment-free

123 approaches can be classified into three main groups. The first group analyzes the frequency of words

124 with a certain length (e.g., FFP (Sims, et al. 2009; Jun, et al. 2010) and CVTree (Zuo and Hao 2015)). The

125 second group matches lengths of overlapping sequences (e.g., ACS (Ulitsky, et al. 2006), KMACS

126 (Leimeister and Morgenstern 2014), and Kr (Haubold, et al. 2009)). The last group calculates

127 informational content between sequences (e.g., Co-phylog (Yi and Jin 2013), FSWM (Leimeister, et al.

128 2017), andi (Haubold, et al. 2015), CAM (Miller, McKinnon, et al. 2019a), and codon pairing (Miller,

129 McKinnon, et al. 2019b)). These techniques are still being developed, and new software packages are 130 constantly updated to recover more robust trees.

132 3. Assessing the Phylogenetic Tree

133 Bootstrapping is a common technique to assess the robustness of a phylogeny by randomly sampling 134 characters with replacement and determining if the recovered phylogenetic tree changes. Proponents of 135 bootstrapping point to its ability to uncover the phylogenetic signal under the noise of phylogenetically 136 uninformative characters. Bootstrapping also has statistical properties that allow a confidence value to 137 be placed on clades (Sanderson 1995). On the other hand, critics of bootstrapping point to the statistical 138 assumptions that are violated in DNA characters because DNA characters cannot be considered 139 independently and identically distributed (Sanderson 1995). Furthermore, a bootstrap proportion is 140 generally unbiased but highly imprecise, meaning the bootstrap number can give high confidence that 141 the data support a clade even if the clade is not real (Hillis and Bull 1993).

\section{Biological Construct of Codon Usage Bias}

144 Phylogenomic studies have recently used codon usage bias to recover species relationships with or 145 without ortholog annotations. Various codon usage biases appear to track speciation events and can 
147 biases affect protein and RNA folding, which affects transcription and translational efficiency, as well as

148 gene expression. Although genetic drift drives global codon usages, the majority of codon usage bias

149 within individual genes is influenced by translational selection (Labella, et al. 2019). Figure 1 outlines

150 how codon biases affect protein levels.

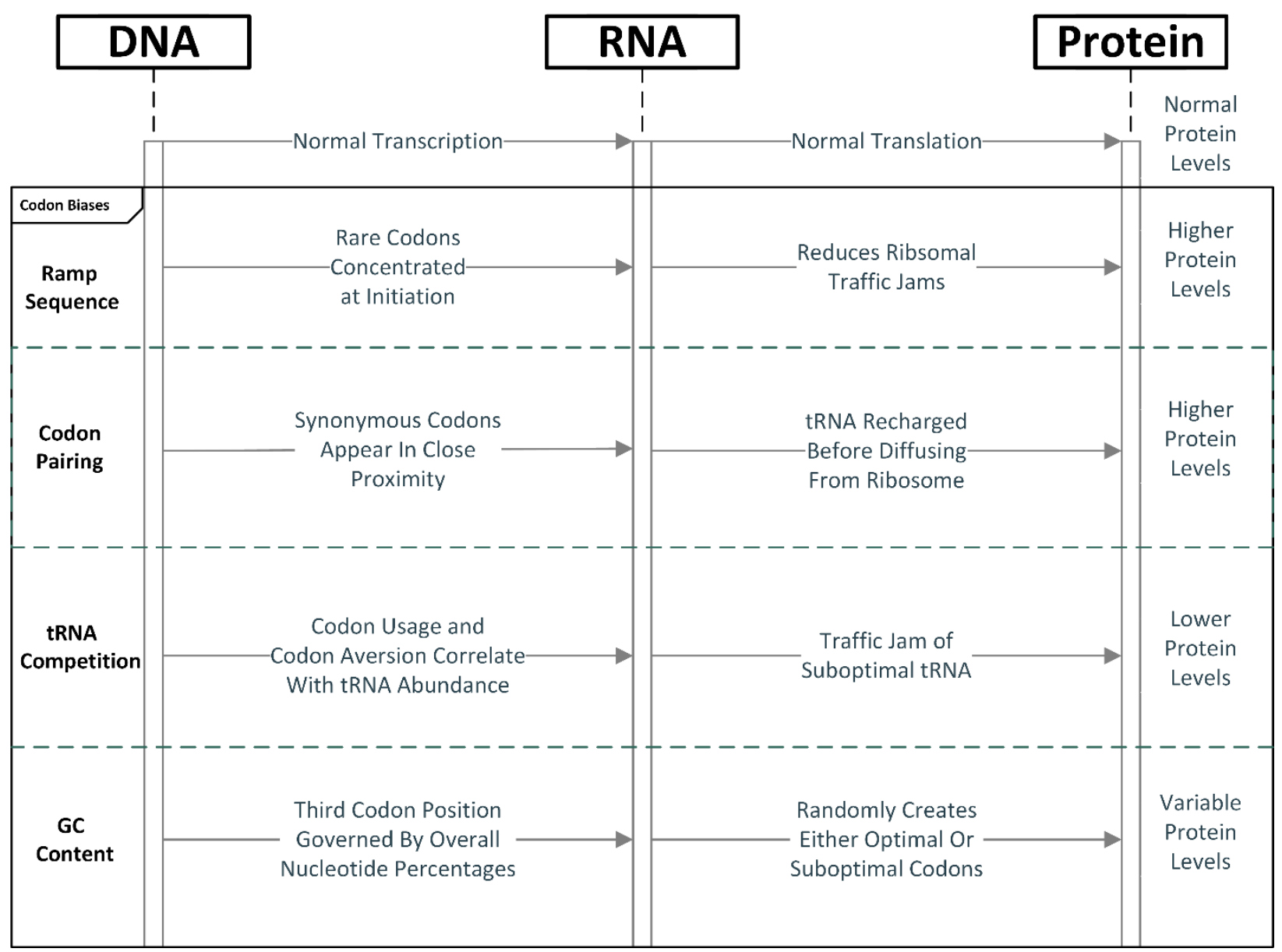

153 Figure 1: How Codon Usage Biases Affect Protein Levels. Many types of codon usage biases directly

154 affect DNA, RNA, and protein secondary structure. They also affect transcription and translational

155 efficiency. The mechanisms by which ramp sequences, codon pairing, tRNA competition, and the GC 156 nucleotide composition affect protein levels are depicted. 
159

160

161

162

163

164

165

166

167

168

169

170

171

172

173

174

175

176

177

178

179

180

181

\section{Codon Usage Metrics}

Several measurements of codon usage preferences facilitate comparing codons. Originally, the Codon Adaptation Index compared the relative codon usage of the most commonly used codons within highly expressed genes (Sharp and Li 1986). Soon thereafter, the effective number of codons quantified the difference in codon usage versus the expected usage if all synonymous codons were used equally (Wright 1990). Because of their simplicity, the effective number of codons and codon adaptation index are still widely used techniques. However, those methods oversimplify the dynamics of codon usage. The tRNA adaptation index (tAl) takes into account the complex relationship between tRNA and codons by using tRNA copy number, gene length, number of codons, and the preponderance of tRNA wobble to determine codon optimality (dos Reis, et al. 2003; dos Reis, et al. 2004). Building on tAl, the normalized translational efficiency (nTE) measurement balances tRNA supply and demand on codon usage and considers cellular tRNA dynamics. A codon is considered "optimal" if the relative supply of its cognate tRNAs exceeds the codon's usage (Pechmann and Frydman 2013). Unfortunately, tAl and nTE require data that are not always available in a species or gene, thus limiting their use across the Tree of Life.

\section{Biological Implications of Codon Usage Bias}

\section{a. Selection toward decreased translational efficiency}

Occasionally, suboptimal codons are more beneficial to cells because they slow translation and allow for more precise, deliberate gene translation. Codon usage bias affects mRNA secondary structure so strongly that local mRNA secondary structure can be used to predict codon usage in highly expressed genes (Trotta 2013). Highly expressed genes also have a ramp of 30-50 slowly-translated, rare codons at the $5^{\prime}$ end of most protein coding sequences (Tuller, et al. 2010) that serves to evenly space ribosomes (Shah, et al. 2013) and reduce mRNA secondary structure (Goodman, et al. 2013) at translation 
182 initiation. A comprehensive analysis of ramp sequences from all domains of life, as well as a method to

183 extract ramp sequences from individual genes is presented in Miller, Brase, et al. (2019).

184

185 Suboptimal codons are also used in genes that are regulated by the cell cycle. Since tRNA expression

186 levels are highest during the $\mathrm{G} 2$ phase, suboptimal codon usage for genes expressed during this phase is

187 also highest. The G1 phase has the lowest tRNA expression, and genes expressed during G1 have a

188 tendency toward optimal codon usage (Frenkel-Morgenstern, et al. 2012).

189

190 Codon usage bias in various bacteria is also associated with species lifestyle (Carbone, et al. 2005;

191 Botzman and Margalit 2011). For cyanobacteria (photosynthetic bacteria), selection toward sub-optimal

192 codon usage produces the circadian clock conditionality, where the circadian clock is expressed only

193 under certain environmental conditions where cyanobacteria are not intrinsically robust (Xu, et al.

194 2013). Similarly, the pathogenicity and habitat of Actinobacteria (High GC gram positive bacteria

195 important for soil systems) also influence codon usage, with aerobic species varying significantly from

196 anaerobic species, and pathogenic species varying significantly from non-pathogenic species (Lal, et al.

197 2016). In each case, codon usage explains bacterial adaptation to their environment.

b. Selection toward increased translational efficiency

200 Highly expressed genes tend to use more optimal codons after the ramp sequence to increase gene

201 translation because optimal codons are translated faster (Quax, et al. 2015). Faster translation is due to

202 decreased wobble interactions, increased optimal tRNA composition, and decreased competition from

203 synonymous codons within a gene. (Brule and Grayhack 2017) Selective pressures for protein expression

204 also act on mRNA sequences to optimize co-translational folding within polypeptides in over $90 \%$ of high

205 expression genes and about 80\% of low expression genes (Pechmann and Frydman 2013). Furthermore, 
206

207 208

209

210

211

212

213

214

215

216

217

218

219

220

221

222

223

224

225

226

227

228

229 gene body methylation is strongly correlated with codon bias, and appears to systematically replace CpG bearing codons, potentially influencing optimal codon establishment (Dixon, et al. 2016).

Recharging a tRNA while the ribosome is still attached to the mRNA strand is another strategy used to increase translational efficiency and decrease overall resource utilization. Co-tRNA codon pairing occurs when two non-identical codons that encode the same amino acid are located in close proximity to each other in a gene. Identical codon pairing occurs when identical codons are located in close proximity in a gene. Co-tRNA and identical codon pairing are mechanisms that a cell uses to reuse a tRNA by recharging the tRNA with an amino acid before the tRNA diffuses, and increases translational speed by approximately 30\% (Cannarozzi, et al. 2010). Although co-tRNA codon pairing occurs more prominently in eukaryotes and identical codon pairing occurs prominently in bacteria (Shao, et al. 2012) and archaea (Zhang, et al. 2013), both co-tRNA and identical codon pairing are phylogenetically conserved in all domains of life (Miller, McKinnon, et al. 2019b).

Other systematic biases also influence codon choice. Background dinucleotide substitution biases from GC to AT and AT to GC often coincide with shifts in optimal codons (Sun, et al. 2017). Even under sustained selective pressure, GC content at the third codon position is highly correlated with overall GC content in a gene, suggesting that optimal codons are affected by overall GC content (Sun, et al. 2017). In an analysis of 65 eukaryotes and prokaryotes, GC content accounted for $76.7 \%$ of amino acid variation (Li, et al. 2015). A summary of mechanisms that affect codon usage bias are shown in Table 1. 
Table 1: Mechanisms Affecting Codon Usage Bias

\begin{tabular}{|c|c|c|}
\hline Name & Location/ Domain & Description \\
\hline Ramp Sequence & $\begin{array}{l}\text { 30-50 nucleotides } \\
\text { downstream of } \\
\text { start codon }\end{array}$ & $\begin{array}{l}\text { The ramp sequence consists of rare, slowly translated } \\
\text { codons that increase ribosomal spacing, reduce mRNA } \\
\text { secondary structure, and slow initial translation. }\end{array}$ \\
\hline Co-tRNA pairing & $\begin{array}{l}\text { More prominent in } \\
\text { eukaryotes. } \\
\text { Phylogenetically } \\
\text { conserved in all } \\
\text { domains of life }\end{array}$ & $\begin{array}{l}\text { tRNA are recharged with amino acids for synonymous } \\
\text { codon translation when synonymous codons are in close } \\
\text { proximity to each other. Recharging allows the tRNA to } \\
\text { stay attached to the ribosome and significantly increases } \\
\text { translation efficiency. }\end{array}$ \\
\hline $\begin{array}{l}\text { Identical Codon } \\
\text { Pairing }\end{array}$ & All domains of life & $\begin{array}{l}\text { tRNA are recharged with amino acids for identical codon } \\
\text { translation when identical codons are in close proximity to } \\
\text { each other. Recharging allows the tRNA to stay attached to } \\
\text { the ribosome and significantly increases translation } \\
\text { efficiency. }\end{array}$ \\
\hline $\begin{array}{l}\text { tRNA } \\
\text { competition }\end{array}$ & $\begin{array}{l}\text { Eukarya, bacteria, } \\
\text { and archaea }\end{array}$ & $\begin{array}{l}\text { Cognate, near-cognate, and non-cognate tRNA may } \\
\text { attempt to bind to an mRNA codon. If relatively few } \\
\text { cognate tRNA are available, translation will slow because } \\
\text { other tRNA attempt to bind to the same codon. This } \\
\text { process is essential for translation elongation, efficiency, } \\
\text { and accuracy (Zur and Tuller 2016). }\end{array}$ \\
\hline GC Content & All domains of life & $\begin{array}{l}\text { Overall GC content in a gene is highly correlated with GC } \\
\text { content at the third codon position. GC content influences } \\
\text { over two-thirds of codon variation. }\end{array}$ \\
\hline
\end{tabular}

\section{Codon Usage Bias in Phylogenetic Systematics}

233 As expected, random mutations are less likely to occur in conserved genomic regions because they can

234 adversely affect fitness, and codon usage bias is less likely to be affected by random mutations than

235 expected based on genomic mutation rates (Castle 2011). Many phylogenetic studies attempt to

236 account for codon usage biases by determining its importance in species relatedness. 


\section{Codon Usage in Maximum Likelihood}

238 Limited codon substitution models have been used for decades in maximum likelihood estimates.

239 However, until recently, a full 61 x 61 codon matrix was too computational intensive to apply to more

240 than a few species and genes (Anisimova and Kosiol 2009). Somewhat surprisingly, after a 61 x 61 codon

241 matrix became computationally viable, it was determined that the full matrix is not always optimal

242 because models that use a fixed codon mutation rate for phylogenetic tree reconstruction fit the data

243 better than a variable codon substitution rate. The apparent variation in codon substitution is actually

244 caused by variable selection against amino acid substitutions in the regions used to develop the model,

245 specifically mitochondria, chloroplast, and hemagglutinin proteins (Miyazawa 2013). Maximum

246 likelihood estimates that use codon models outperform a parsimony analysis only when codon usage is

247 highly skewed and is not affected by asymmetry in substitution rates (approach validated using

248 Drosophila) (Akashi, et al. 2007).

Because full codon models are computationally intensive and do not always elucidate more information than simpler models, common likelihood approaches use nonsynonymous to synonymous mutation

252 rates per site $\left(d_{N} / d_{s}\right)$ instead of the complete codon model. If the codon usage bias is strongly

253 conserved, then $d_{s}$ will decrease and $d_{N} / d_{S}$ will increase within a population. The $d_{N} / d_{S}$ ratio was used in

254 Drosophila lineages, and helped determine that the Notch locus had evolved to include suboptimal

255 codons (Nielsen, et al. 2007). Using 158 orthologous genes, maximum likelihood also detected a strong

256 shift from suboptimal to optimal codons in two lineages of Populus (Ingvarsson 2008). Detecting the

257 cause of such shifts in codon usage is important for determining the biological significance of mutations.

258 SCUMBLE (Synonymous Codon Usage Bias Maximum Likelihood Estimation) uses a model inspired by

259 statistical physics to identify different sources of codon bias including selection and mutation (Kloster

260 and Tang 2008). SCUMBLE is also used as a filter to identify regions with insufficient information for 
261 analysis. This technique helped determine that natural selection shaped codon biases in

262 Strongylocentrotus purpuratus (purple sea urchin) by limiting the analysis to only regions with sufficient

263 support (Kober and Pogson 2013). Shifts in mutation and selection rates allow the evolutionary history

264 of species to be recovered using this method.

265

266

2. Violations of Maximum Likelihood Statistical Properties in a Codon Model

267 Many of the assumptions of the statistical properties in maximum likelihood are violated by a codon

268 model. For instance, species are constrained to taxon-specific pools of tRNA, and triplets in coding

269 sequences are not independent. Algorithms with statistical properties that require character

270 independence, such as maximum likelihood, violate that rule for genetic data (Christianson 2005).

271 Furthermore, the codon model assumption of homogeneity of codon composition leads to seriously

272 biased phylogenetic estimations when that assumption is violated (Inagaki and Roger 2006).

273

274 Horizontal gene transfer is another important mechanism in evolution and complicates phylogenetic

275 analyses in bacteria because $81 \pm 15 \%$ of genes have been laterally transferred among bacteria at some

276 point in their evolutionary history (Dagan, et al. 2008). Common transposable elements in eukaryotes

277 also arose from horizontal gene transfer, with over $50 \%$ of some mammalian genomes originally arising

278 from horizontal gene transfer (Ivancevic, et al. 2018). Detecting horizontal gene transfer has been

279 challenging, and codon bias is a poor indicator of horizontal transmission, normally underestimating the

280 effects of lateral transfer (Koski, et al. 2001; Tuller 2011; Friedman and Ely 2012). However, codon

281 composition is an excellent indicator of whether a gene will become fixed in a species after a lateral

282 transfer event (Tuller 2011). The concept of horizontal gene transfer not only complicates a general

283 phylogenetic analysis, but suggests that a standard bifurcating tree might not be the best choice in

284 analyses of bacteria or archaea (Koonin and Wolf 2008). Although it is known that codons (and DNA in 
285

286

287

288

289

290

291

292

293

294

295

296

297

298

299

300

301

302

303

304

305

306

307

308

general) do not strictly follow many of the assumptions of phylogenetic analyses, the bifurcating tree is still the most widely used phylogenetic representation, and generally depicts statements of homology even when some assumptions are violated.

\section{Codon Usage in Viruses}

Another purpose of phylogenies is to predict the pathogenicity of viruses and viral interactions with their hosts. Bee-infecting viruses have strong correlations in their codon usages with their hosts, and the infected insects' codon usage similarity follows the insect phylogeny (Chantawannakul and Cutler 2008). Furthermore, human-host viruses tend to share the same codon usages as proteins expressed in tissues that the viruses infect (Miller, Hippen, Wright, et al. 2017). More specifically, the key determinant in codon patterns within herpesviruses were the overall GC content, GC content at the $3^{\text {rd }}$ codon position, and gene length (Roychoudhury and Mukherjee 2010). In contrast, mutation played a larger role in Zika viruses, with higher frequencies of A-ending codons (Cristina, et al. 2016). However, evidence of natural selection in Zika viruses also suggest that they evolved host- and vector-specific codon usage patterns to successfully replicate in various hosts and vectors (Butt, et al. 2016). In hepatitis C, preferred codon usages did not always match the phylogenetic histories of the viruses as determined by sequence similarity, indicating that codon usage might provide additional information not identified in common phylogenomic approaches (Mortazavi, et al. 2016).

\section{Successful Implementations of Codon Usage Bias in Phylogenetics}

Beyond analyzing pathogenicity, phylogenetic inferences using codon usage bias from all domains of life have successfully uncovered several interesting biological principles. One study found compositional differences in codon usage between monocots (flowering plants whose seeds contain one embryonic leaf) and dicots (flowering plants whose seeds contains two embryonic leaves), where monocots had 
309

310

311

312

313

314

315

316

317

318

319

320

321

322

323

324

325

326

327

328

329

330

331

332

lower DNA background compositional bias, but higher codon usage bias than dicots (Camiolo, et al. 2015). Another technique used a distance-based clustering method of codon usage weighted by nucleotide base bias per position (i.e., the frequency of a codon over the product of the frequency of the nucleotide at the first, second, and third positions) to recover the phylogeny of closely related Ectocarpales (brown algae) (Das, et al. 2005). The phylogenetic signal of codon usage was not limited to nuclear DNA, and mitochondrial synonymous codon usage in plants was associated with intron number that mirrored species evolution (Xu, et al. 2015).

Creative attempts at analyzing codon usage have also proven fruitful. A binary representation of codon aversion (i.e., creating a character matrix based on codons which are not used in an ortholog) successfully recover the phylogeny of various tetrapods, showing that complete codon aversion is also conserved (Miller, Hippen, Belyeu, et al. 2017). That study also found that stop codon usage had the highest phylogenetic signal (Miller, Hippen, Belyeu, et al. 2017), meaning a codon matrix of $64 \times 64$ (the probability of all codons including the stop codons transitioning to all other codons) might be better than the traditional $61 \times 61$ codon matrix in a likelihood framework. Codon aversion has also been used in an alignment-free context by comparing sets of codon tuples found in a genome, where each tuple is a list of codons not used in a gene (Miller, McKinnon, et al. 2019a). A similar technique used codon pairing and codon pairs (i.e., the same codon being used within a ribosomal window) and was phylogenetically informative in both alignment-free and parsimony frameworks (Miller, McKinnon, et al. 2019b).

Other studies map codon usage in a particular gene across a reference phylogeny. This technique can produce meaningful representations of codon transitions across genes. Mapping the codon usage bias of a gene tree to a species tree revealed purifying selection among the actin-depolymerizing factor/cofilin 
333 (ADF/CFL) gene family (Roy-Zokan, et al. 2015). This technique also showed that codon usage is

334 significantly correlated with gene age within metazoan genomes (Prat, et al. 2009). Codon aversion in all 335 domains of life was also mapped to the Open Tree of Life (OTL) (Hinchliff, et al. 2015) and showed that 336 codon aversion follows established species relationships more closely than expected by random chance 337 (Miller, McKinnon, et al. 2019c).

\section{Concluding Remarks}

340 Codon usage bias continues to be widely studied in a phylogenetic construct. However, its application in 341 phylogenomics remains limited by its applicability in current phylogenomic techniques. While some 342 applications attempt to incorporate codon usage bias as a novel character state in phylogenetics or in a 343 maximum likelihood framework, many of the key attributes of codon bias remain unexplored. For 344 instance, although the ramp of slowly translated codons has been identified, it is unknown if the ramp 345 sequence is more or less phylogenetically conserved than the rest of the gene sequence.

347 In addition, although it is known that tRNA supply and demand is correlated to codon usage, a model 348 does not currently exist to assess tRNA supply and demand in a maximum likelihood framework. Future codon analyses will necessitate more complete datasets with accurate tRNA expression values in

350 different tissues and species. A more robust dataset of tRNA expression values would also facilitate 351 codon model analyses. Furthermore, since codons are used to regulate gene translational efficiency, 352 codon models might require gene expression data in addition to the full (or reduced) codon matrix.

354 Codon usage bias is an exciting biological principle that has not been fully utilized in phylogenetic 355 systematics. Few likelihood methods use codon bias, and many aspects of the ramp sequence, co-tRNA 356 codon pairing, gene expression, and tRNA expression have yet to be explored. Although codon usage 
357 bias has been shown to be phylogenetically conserved, many of the biological principles surrounding

358 codon usage bias have yet to be fully utilized in phylogenomics. We propose that more research into

359 codon usage bias and its phylogenetic conservation will be beneficial to future phylogenomic studies by

360 providing researchers with more robust phylogenetic trees.

\section{Acknowledgements}

363 We appreciate the continued support of Brigham Young University.

\section{Authors' Contributions}

JM and PR conceived the idea. JM led the writing of the manuscript. All authors contributed critically to

367 the drafts, edited the drafts, and gave final approval for publication.

\section{References}

Akashi H, Goel P, John A. 2007. Ancestral inference and the study of codon bias evolution: implications

371 for molecular evolutionary analyses of the Drosophila melanogaster subgroup. PLoS One 2:e1065.

372 Anisimova M, Kosiol C. 2009. Investigating protein-coding sequence evolution with probabilistic codon

373 substitution models. Mol Biol Evol 26:255-271.

374 Bertolani R, Guidetti R, Marchioro T, Altiero T, Rebecchi L, Cesari M. 2014. Phylogeny of Eutardigrada:

375 New molecular data and their morphological support lead to the identification of new evolutionary

376 lineages. Molecular Phylogenetics and Evolution 76:110-126.

377 Botzman M, Margalit H. 2011. Variation in global codon usage bias among prokaryotic organisms is 378 associated with their lifestyles. Genome Biol 12:R109.

379 Bouckaert R, Heled J, Kuhnert D, Vaughan T, Wu CH, Xie D, Suchard MA, Rambaut A, Drummond AJ.

380 2014. BEAST 2: a software platform for Bayesian evolutionary analysis. PLoS Comput Biol 10:e1003537. 
381 382 383 384

Brule CE, Grayhack EJ. 2017. Synonymous Codons: Choose Wisely for Expression. Trends Genet 33:283297.

Butt AM, Nasrullah I, Qamar R, Tong Y. 2016. Evolution of codon usage in Zika virus genomes is host and vector specific. Emerging Microbes \&Amp; Infections 5:e107.

Camiolo S, Melito S, Porceddu A. 2015. New insights into the interplay between codon bias determinants in plants. DNA Research:dsv027.

Cannarozzi G, Schraudolph NN, Faty M, von Rohr P, Friberg MT, Roth AC, Gonnet P, Gonnet G, Barral Y. 2010. A role for codon order in translation dynamics. Cell 141:355-367.

Carbone A, Kepes F, Zinovyev A. 2005. Codon bias signatures, organization of microorganisms in codon space, and lifestyle. Mol Biol Evol 22:547-561.

Castle JC. 2011. SNPs occur in regions with less genomic sequence conservation. PLoS One 6:e20660.

Chan CX, Bernard G, Poirion O, Hogan JM, Ragan MA. 2014. Inferring phylogenies of evolving sequences without multiple sequence alignment. Sci Rep 4:6504.

Chantawannakul P, Cutler RW. 2008. Convergent host-parasite codon usage between honeybee and bee associated viral genomes. J Invertebr Pathol 98:206-210.

Christianson ML. 2005. Usuage patterns distort phylogenies from or of DNA sequences. 92:1221-1233.

Crick FH. 1966. Codon--anticodon pairing: the wobble hypothesis. J Mol Biol 19:548-555.

Crick FH, Barnett L, Brenner S, Watts-Tobin RJ. 1961. General nature of the genetic code for proteins. Nature 192:1227-1232.

Cristina J, Fajardo A, Sonora M, Moratorio G, Musto H. 2016. A detailed comparative analysis of codon usage bias in Zika virus. Virus Res 223:147-152.

Dagan T, Artzy-Randrup Y, Martin W. 2008. Modular networks and cumulative impact of lateral transfer in prokaryote genome evolution. Proceedings of the National Academy of Sciences 105:10039-10044. 
404

405

406

407 Daugelaite J, O' Driscoll A, Sleator RD. 2013. An Overview of Multiple Sequence Alignments and Cloud

408 Computing in Bioinformatics. ISRN Biomathematics 2013:14.

409 Dixon GB, Bay LK, Matz MV. 2016. Evolutionary Consequences of DNA Methylation in a Basal Metazoan.

410

411 dos Reis M, Savva R, Wernisch L. 2004. Solving the riddle of codon usage preferences: a test for

412 translational selection. Nucleic Acids Res 32:5036-5044.

413 dos Reis M, Wernisch L, Savva R. 2003. Unexpected correlations between gene expression and codon

414 usage bias from microarray data for the whole Escherichia coli K-12 genome. Nucleic Acids Res 31:6976-

4156985.

416 Edgar RC. 2004. MUSCLE: multiple sequence alignment with high accuracy and high throughput. Nucleic

417 Acids Res 32:1792-1797.

418 Farris JS. 2008. Parsimony and explanatory power. Cladistics 24:825-847.

419 Felsenstein J. 1978. Cases in which Parsimony or Compatibility Methods will be Positively Misleading.

420 Systematic Biology 27:401-410.

421 Frenkel-Morgenstern M, Danon T, Christian T, Igarashi T, Cohen L, Hou Y-M, Jensen LJ. 2012. Genes

422 adopt non-optimal codon usage to generate cell cycle-dependent oscillations in protein levels.

423 Molecular Systems Biology 8:572-572.

424 Friedman R, Ely B. 2012. Codon usage methods for horizontal gene transfer detection generate an

425 abundance of false positive and false negative results. Curr Microbiol 65:639-642.

426 Goloboff PA, Farris JS, Nixon KC. 2005. TNT: Tree Analysis Using New Technology. 54:176-178. 
427 Goodman DB, Church GM, Kosuri S. 2013. Causes and effects of N-terminal codon bias in bacterial 428 genes. Science 342:475-479.

429 Gutman GA, Hatfield GW. 1989. Nonrandom utilization of codon pairs in Escherichia coli. Proc Natl Acad 430 Sci U S A 86:3699-3703.

431 Haszprunar G. 1992. The types of homology and their significance for evolutionary biology and 432 phylogenetics. Journal of Evolutionary Biology 5:13-24.

433 Haubold B, Klotzl F, Pfaffelhuber P. 2015. andi: fast and accurate estimation of evolutionary distances 434 between closely related genomes. Bioinformatics 31:1169-1175.

435 Haubold B, Pfaffelhuber P, Domazet-Loso M, Wiehe T. 2009. Estimating mutation distances from 436 unaligned genomes. J Comput Biol 16:1487-1500.

437 Hillis DM, Bull JJ. 1993. An Empirical Test of Bootstrapping as a Method for Assessing Confidence in 438 Phylogenetic Analysis. Systematic Biology 42:182-192.

439 Hinchliff CE, Smith SA, Allman JF, Burleigh JG, Chaudhary R, Coghill LM, Crandall KA, Deng J, Drew BT, 440 Gazis R, et al. 2015. Synthesis of phylogeny and taxonomy into a comprehensive tree of life. Proc Natl $441 \quad$ Acad Sci U S A 112:12764-12769.

442 Holder M, Lewis PO. 2003. Phylogeny estimation: traditional and Bayesian approaches. Nat Rev Genet $443 \quad 4: 275-284$.

444 Huelsenbeck JP, Larget B, Miller RE, Ronquist F. 2002. Potential applications and pitfalls of Bayesian 445 inference of phylogeny. Syst Biol 51:673-688.

446 Inagaki Y, Roger AJ. 2006. Phylogenetic estimation under codon models can be biased by codon usage 447 heterogeneity. Mol Phylogenet Evol 40:428-434.

448 Ingvarsson PK. 2008. Molecular evolution of synonymous codon usage in Populus. BMC Evol Biol 8:307.

449 Ivancevic AM, Kortschak RD, Bertozzi T, Adelson DL. 2018. Horizontal transfer of BovB and L1

450 retrotransposons in eukaryotes. Genome Biol 19:85. 
451 Jun S-R, Sims GE, Wu GA, Kim S-H. 2010. Whole-proteome phylogeny of prokaryotes by feature

452 frequency profiles: An alignment-free method with optimal feature resolution. Proceedings of the

453 National Academy of Sciences 107:133-138.

454 Katoh K, Standley DM. 2014. MAFFT: iterative refinement and additional methods. Methods Mol Biol

$455 \quad$ 1079:131-146.

456 Kloster M, Tang C. 2008. SCUMBLE: a method for systematic and accurate detection of codon usage bias

457 by maximum likelihood estimation. Nucleic Acids Res 36:3819-3827.

458 Kober KM, Pogson GH. 2013. Genome-Wide Patterns of Codon Bias Are Shaped by Natural Selection in

459 the Purple Sea Urchin, \&lt;em\&gt;Strongylocentrotus purpuratus\&lt;/em\&gt. G3:

460 Genes|Genomes|Genetics 3:1069.

461 Koonin EV. 2005. Orthologs, paralogs, and evolutionary genomics. Annu Rev Genet 39:309-338.

462 Koonin EV, Wolf YI. 2008. Genomics of bacteria and archaea: the emerging dynamic view of the

463 prokaryotic world. Nucleic Acids Research 36:6688-6719.

464 Koski LB, Morton RA, Golding GB. 2001. Codon bias and base composition are poor indicators of

465 horizontally transferred genes. Mol Biol Evol 18:404-412.

466 Kumar S, Stecher G, Li M, Knyaz C, Tamura K. 2018. MEGA X: Molecular Evolutionary Genetics Analysis

467 across Computing Platforms. Mol Biol Evol 35:1547-1549.

468 Labella AL, Opulente DA, Steenwyk JL, Hittinger CT, Rokas A. 2019. Variation and selection on codon

469 usage bias across an entire subphylum. PLOS Genetics 15:e1008304.

470 Lal D, Verma M, Behura SK, Lal R. 2016. Codon usage bias in phylum Actinobacteria : relevance to

471 environmental adaptation and host pathogenicity. Research in Microbiologoy 167:669-677.

472 Leimeister CA, Morgenstern B. 2014. Kmacs: the k-mismatch average common substring approach to

473 alignment-free sequence comparison. Bioinformatics 30:2000-2008. 
474 Leimeister CA, Sohrabi-Jahromi S, Morgenstern B. 2017. Fast and accurate phylogeny reconstruction

475 using filtered spaced-word matches. Bioinformatics 33:971-979.

476 Li J, Zhou J, Wu Y, Yang S, Tian D. 2015. GC-Content of Synonymous Codons Profoundly Influences Amino

477 Acid Usage. G3 (Bethesda) 5:2027-2036.

478 Ling C, Hamada T, Gao J, Zhao G, Sun D, Shi W. 2016. MrBayes tgMC3++: A High Performance and

479 Resource-Efficient GPU-Oriented Phylogenetic Analysis Method. IEEE/ACM Trans Comput Biol Bioinform $480 \quad 13: 845-854$.

481 Magis C, Taly JF, Bussotti G, Chang JM, Di Tommaso P, Erb I, Espinosa-Carrasco J, Notredame C. 2014. T-

482 Coffee: Tree-based consistency objective function for alignment evaluation. Methods Mol Biol 1079:117-

483129.

484 Miller JB, Brase LR, Ridge PG. 2019. ExtRamp: a novel algorithm for extracting the ramp sequence based 485 on the tRNA adaptation index or relative codon adaptiveness. Nucleic Acids Res.

486 Miller JB, Hippen AA, Belyeu JR, Whiting MF, Ridge PG. 2017. Missing something? Codon aversion as a

487 new character system in phylogenetics. Cladistics:n/a-n/a.

488 Miller JB, Hippen AA, Wright SM, Morris C, Ridge PG. 2017. Human viruses have codon usage biases that 489 match highly expressed proteins in the tissues they infect. Biomedical Genetics and Genomics 2.

490 Miller JB, McKinnon LM, Whiting MF, Ridge PG. 2019a. CAM: An alignment-free method to recover 491 phylogenies using codon aversion motifs. PeerJ Preprints 7:e27756v27751.

492 Miller JB, McKinnon LM, Whiting MF, Ridge PG. 2019b. Codon Pairs are Phylogenetically Conserved:

493 Codon pairing as a new class of phylogenetic characters. bioRxiv:654947.

494 Miller JB, McKinnon LM, Whiting MF, Ridge PG. 2019c. Codon Use and Aversion is Largely

495 Phylogenetically Conserved Across the Tree of Life. bioRxiv:649590.

496 Miyazawa S. 2013. Superiority of a mechanistic codon substitution model even for protein sequences in

497 Phylogenetic analysis.1-10. 
498

499

500

501

502

503

504

505

506

507

508

509

510

511

512

513

514

515

516

517

518

519

520

Mortazavi M, Zarenezhad M, Alavian SM, Gholamzadeh S, Malekpour A, Ghorbani M, Torkzadeh Mahani M, Lotfi S, Fakhrzad A. 2016. Bioinformatic Analysis of Codon Usage and Phylogenetic Relationships in Different Genotypes of the Hepatitis C Virus. Hepatitis Monthly 16:e39196.

Nguyen LT, Schmidt HA, von Haeseler A, Minh BQ. 2015. IQ-TREE: a fast and effective stochastic algorithm for estimating maximum-likelihood phylogenies. Mol Biol Evol 32:268-274.

Nielsen R, Bauer DuMont VL, Hubisz MJ, Aquadro CF. 2007. Maximum likelihood estimation of ancestral codon usage bias parameters in Drosophila. Mol Biol Evol 24:228-235.

Pais FS, Ruy Pde C, Oliveira G, Coimbra RS. 2014. Assessing the efficiency of multiple sequence alignment programs. Algorithms Mol Biol 9:4.

Paninski L, Pillow JW, Simoncelli EP. 2004. Maximum likelihood estimation of a stochastic integrate-andfire neural encoding model. Neural Comput 16:2533-2561.

Pechmann S, Frydman J. 2013. Evolutionary conservation of codon optimality reveals hidden signatures of cotranslational folding. Nat Struct Mol Biol 20:237-243.

Philippe H, Brinkmann H, Lavrov DV, Littlewood DTJ, Manuel M, Wörheide G, Baurain D. 2011. Resolving Difficult Phylogenetic Questions: Why More Sequences Are Not Enough. PLOS Biology 9:e1000602.

Post LE, Strycharz GD, Nomura M, Lewis H, Dennis PP. 1979. Nucleotide sequence of the ribosomal protein gene cluster adjacent to the gene for RNA polymerase subunit beta in Escherichia coli. Proc Natl Acad Sci U S A 76:1697-1701.

Prat Y, Fromer M, Linial N, Linial M. (Prat2009 co-authors). 2009. Codon usage is associated with the evolutionary age of genes in metazoan genomes. BMC Evolutionary Biology 9:285.

Quax TE, Claassens NJ, Soll D, van der Oost J. 2015. Codon Bias as a Means to Fine-Tune Gene Expression. Mol Cell 59:149-161.

Retief JD. 2000. Phylogenetic analysis using PHYLIP. Methods Mol Biol 132:243-258. 
521 Rogers JS. 1997. On the consistency of maximum likelihood estimation of phylogenetic trees from

522 nucleotide sequences. Syst Biol 46:354-357.

523 Ronquist F, Teslenko M, van der Mark P, Ayres DL, Darling A, Hohna S, Larget B, Liu L, Suchard MA,

524 Huelsenbeck JP. 2012. MrBayes 3.2: efficient Bayesian phylogenetic inference and model choice across a

525 large model space. Syst Biol 61:539-542.

526 Roy-Zokan EM, Dyer KA, Meagher RB. 2015. Phylogenetic Patterns of Codon Evolution in the ACTIN-

527 DEPOLYMERIZING FACTOR/COFILIN (ADF/CFL) Gene Family. PLoS One 10:e0145917.

528 Roychoudhury S, Mukherjee D. 2010. A detailed comparative analysis on the overall codon usage

529 pattern in herpesviruses. Virus Res 148:31-43.

530 Sanderson MJ. 1995. Objections to Bootstrapping Phylogenies: A Critique. Systematic Biology 44:299-

531320.

532 Shah P, Ding Y, Niemczyk M, Kudla G, Plotkin JB. 2013. Rate-limiting steps in yeast protein translation.

533 Cell 153:1589-1601.

534 Shao ZQ, Zhang YM, Feng XY, Wang B, Chen JQ. 2012. Synonymous codon ordering: a subtle but

535 prevalent strategy of bacteria to improve translational efficiency. PLoS One 7:e33547.

536 Sharp PM, Li WH. 1986. An evolutionary perspective on synonymous codon usage in unicellular

537 organisms. J Mol Evol 24:28-38.

538 Siddall ME. 1998. Success of Parsimony in the Four-Taxon Case: Long-Branch Repulsion by Likelihood in

539 the Farris Zone. Cladistics 14:209-220.

540 Sievers F, Higgins DG. 2014. Clustal omega. Curr Protoc Bioinformatics 48:3 13 11-16.

541 Sievers F, Higgins DG. 2018. Clustal Omega for making accurate alignments of many protein sequences.

542 Protein Sci 27:135-145.

543 Sims GE, Jun SR, Wu GA, Kim SH. 2009. Alignment-free genome comparison with feature frequency

544 profiles (FFP) and optimal resolutions. Proc Natl Acad Sci U S A 106:2677-2682. 
545 Soltis DE, Soltis PS. 2003. The Role of Phylogenetics in Comparative Genetics. Plant Physiology 132:1790-

5461800.

547 Stamatakis A. 2014. RAxML version 8: a tool for phylogenetic analysis and post-analysis of large

548 phylogenies. Bioinformatics 30:1312-1313.

549 Sun Y, Tamarit D, Andersson SGE. 2017. Switches in Genomic GC Content Drive Shifts of Optimal Codons

550 under Sustained Selection on Synonymous Sites. Genome Biol Evol 9:2560-2579.

551 Tekaia F. 2016. Inferring Orthologs: Open Questions and Perspectives. Genomics Insights 9:17-28.

552 Trotta E. 2013. Selection on codon bias in yeast: a transcriptional hypothesis. Nucleic Acids Res 41:9382-

5539395.

554 Tuller T. 2011. Codon bias, tRNA pools and horizontal gene transfer. Mob Genet Elements 1:75-77.

555 Tuller T, Carmi A, Vestsigian K, Navon S, Dorfan Y, Zaborske J, Pan T, Dahan O, Furman I, Pilpel Y. 2010.

556 An evolutionarily conserved mechanism for controlling the efficiency of protein translation. Cell

$557 \quad 141: 344-354$.

558 Ulitsky I, Burstein D, Tuller T, Chor B. 2006. The average common substring approach to phylogenomic

559 reconstruction. J Comput Biol 13:336-350.

560 Wald A. 1949. Note on the Consistency of the Maximum Likelihood Estimate.595-601.

561 Wilgenbusch JC, Swofford D. 2003. Inferring evolutionary trees with PAUP*. Curr Protoc Bioinformatics

562 Chapter 6:Unit 64.

563 Wright F. 1990. The 'effective number of codons' used in a gene. Gene 87:23-29.

564 Xu W, Xing T, Zhao M, Yin X, Xia G, Wang M. 2015. Synonymous codon usage bias in plant mitochondrial 565 genes is associated with intron number and mirrors species evolution. PLoS One 10:e0131508.

566 Xu Y, Ma P, Shah P, Rokas A, Liu Y, Johnson CH. 2013. Non-optimal codon usage is a mechanism to 567 achieve circadian clock conditionality. Nature 495:116-120.

568 Yang Z, Rannala B. 2012. Molecular phylogenetics: principles and practice. Nat Rev Genet 13:303-314. 
569 Yi H, Jin L. 2013. Co-phylog: an assembly-free phylogenomic approach for closely related organisms.

$570 \quad$ Nucleic Acids Res 41:e75.

571 Zhang YM, Shao ZQ, Yang LT, Sun XQ, Mao YF, Chen JQ, Wang B. 2013. Non-random arrangement of

572 synonymous codons in archaea coding sequences. Genomics 101:362-367.

573 Zuo G, Hao B. 2015. CVTree3 Web Server for Whole-genome-based and Alignment-free Prokaryotic

574 Phylogeny and Taxonomy. Genomics Proteomics Bioinformatics 13:321-331.

575 Zur H, Tuller T. 2016. Predictive biophysical modeling and understanding of the dynamics of mRNA

576 translation and its evolution. Nucleic Acids Research 44:9031-9049.

577 